Chapter 26.

\title{
Specialty Asian Vegetable Production in South Florida
}

\section{M.L. Lamberts}

The term "specialty Asian vegetables" is used herein to designate a group of lesser known vegetables which are primarily Asian in origin. At present, most consumers who purchase these products are either Asian or are preparing Asian cuisine. Some may have potential for appealing to a wider range of consumers.

\section{CUCURBITS}

This group (Table 1) includes fruits, eaten either immature or mature, and several vegetables with edible tender stems and leaves. All can be grown on raised beds using plastic mulch and drip irrigation. Fertilizer recommendations for cucumbers are applicable to bitter melon (Fig. 261), smooth loofah, Chinese okra, and bottle gourd (Table 2 and Table 3). Recommendations for watermelons should be followed for winter melon. No recommendations exist for chayote (Fig. 26-2), tindora (Fig. 26-3) or parvar, though a program comparable to that for watermelon would most closely approximate the nutritional needs of these crops (Table 2 and Table 3). With the exception of tindora and parvar which are propagated by stem cuttings, all can be started from seed or transplants (Table 4). Many need some type of support for quality fruit production (Table 4).

\section{LEGUMES}

The Asian legume group (Table 5) includes fruits (usually known as pods), which are eaten at the immature stage plus some crops with edible immature seeds. The winged bean (Fig. 26-4) also has edible leaves and roots, though the latter do not appear to be cultivated commercially in the Continental U.S. All the pole or indeterminate types can be grown on raised beds using plastic mulch and drip irrigation.The determinate types (yard long bean and lablab bean) can also be grown on plastic mulch,but the growing season may be too short to justify this expense. Fertilizer recommendations for pole beans are applicable to this group (Table 6). All of these crops should be started from seed, with growers taking note that winged beans require scarification prior to planting (Table 7). All the indeterminate types need some type of support (Table 7).

\section{SOLANUMS}

The group (Table 8) includes fruits which are eaten at the immature or mature stage. Pea eggplant (Fig. 26-5) also has edible tender shoots. All can be grown on raised beds using plastic mulch and drip irrigation. Fertilizer recommendations for eggplant should be used for the Thai (Fig. 26-6), Japanese and pea eggplant, while recommendations for pepper should be followed for bird's eye peppers (Table 9 and Table 10). These crops can be started from seed or transplants (Table 11). All the indeterminate types need some type of support (Table 11).

\section{OTHER}

The final group (Table 12) includes fruits, which are eaten at the immature stage and edible leaves or stem tips. With the exception of sweetpotato, no fertilizer recommendations have been developed for this group. These crops should be started from seed, corms or stem cuttings (Table 13).

\section{CONCLUSIONS}

The specialty Asian vegetables grown in south Florida are shipped to Asian markets, with very few being sold in "traditional" supermarkets. Yields vary by crop (Table 14), ranging from $2,400 \mathrm{lbs} /$ acre for smooth loofah and winged beans to $13,500 \mathrm{lbs} /$ acre for fuzzy squash and long squash. Growers are primarily Asians, and even this group has noted some difficulties in selling to markets other than their particular nationality or ethnic group. Anyone considering producing any of these crops should do a thorough study of the prospective market before planting. Experimental plantings should be very limited in size since even minute changes in volume can have drastic effects on market price. As with other commodities grown in Florida, this group experiences competition with off-shore production, primarily from the Caribbean. 
Table 1. Common and scientific names, synonyms, and edible portions of Asian cucurbits.

\begin{tabular}{||llll||}
\hline Common Name & Scientific Name & Synonyms & Edible Portion \\
\hline \hline bitter melon & Momordica charantia L. & bitter gourd, balsam pear & immature / mature fruit \\
winter melon & Benincasa hispida (Thunb.) Cogn. & wax gourd & immature / mature fruit \\
smooth loofah & Luffa aegyptiaca Miller & dishcloth or sponge gourd & immature fruit \\
Chinese okra & Luffa acutangula (L.) Roxb. & angled loofah & immature fruit, leaves \\
long squash & Laginaria siceraria (Mol.) Standl. & calabash or bottle gourd & immature fruit, tender shoots, leaves \\
chayote & Sechium edule (Jacq.) Swartz & mirilton, vegetable pear & fruit, tender shoots, leaves \\
tindora & Coccinia grandis (L.) Voigt & ivy gourd & fruit, tender shoots, leaves \\
parvar & Tricosanthes dioica Roxb. & pointed gourd & immature fruit, tender shoots \\
\hline \hline 1 Italics indicate the edible portion grown in south Florida & & \\
\hline
\end{tabular}

Table 2. Target $\mathrm{pH}$, and recommended $\mathrm{N}, \mathrm{P}_{2} \mathrm{O}_{5}$, and $\mathrm{K}_{2} \mathrm{O}$ fertilizer for specialty Asian cucurbits.

\begin{tabular}{|c|c|c|c|c|c|c|c|c|c|c|c|}
\hline \multirow[t]{2}{*}{ Target pH } & $\mathrm{N} \mathrm{Ib/A}$ & VL & L & M & H & VH & VL & L & M & $\mathrm{H}$ & VH \\
\hline & & \multicolumn{4}{|c|}{$\mathrm{P}_{2} \mathrm{O}_{5}$} & $(\mathrm{Ib} / \mathrm{A} / \mathrm{c}$ & \multicolumn{5}{|c|}{$\mathrm{K}_{2} \mathrm{O}$} \\
\hline \multicolumn{12}{|c|}{ bitter melon, smooth loofah, Chinese okra, long squash } \\
\hline \multicolumn{11}{|c|}{ winter melon, chayote tips, tindora, parvar } & 0 \\
\hline 6.5 & 150 & 150 & 120 & 80 & 0 & 0 & 150 & 120 & 80 & 0 & 0 \\
\hline
\end{tabular}

Table 3. Injection schedule for $\mathrm{N}$ and $\mathrm{K}$ for specialty Asian cucurbit crops grown on soils testing very low in $\mathrm{K}$.

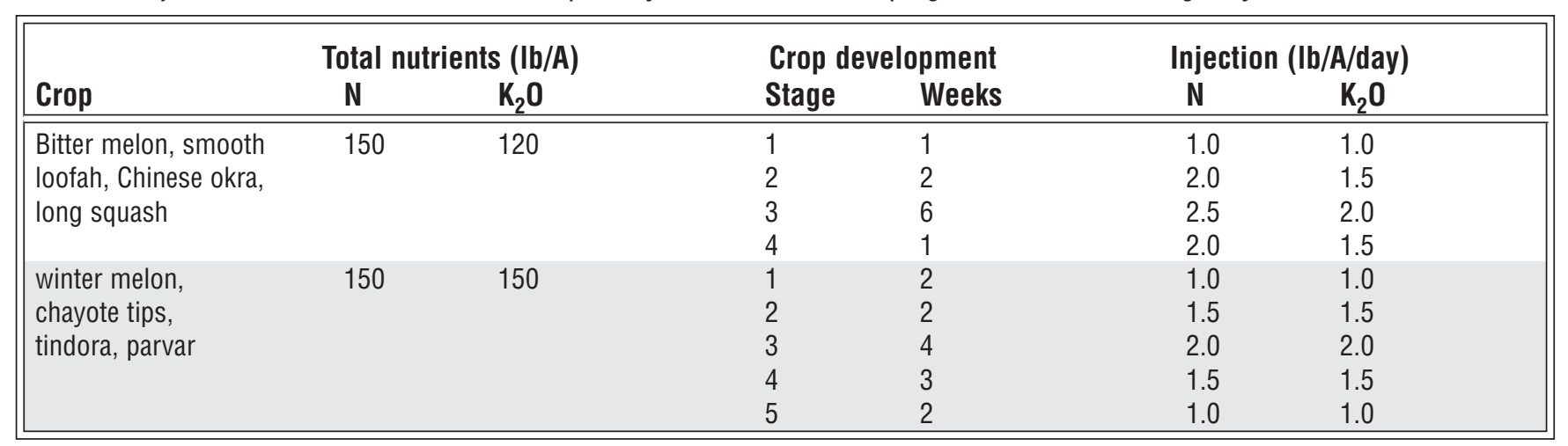

Table 4. Life cycle, season, trellising requirements, mulching, and planting material for Asian cucurbits.

\begin{tabular}{|c|c|c|c|c|c|}
\hline Crop & Life-cycle & Season & Trellis & Mulch & Planting Material \\
\hline bitter melon & annual & fall-spring & yes & yes & seed \\
\hline winter melon & annual & fall-spring & no & yes & seed \\
\hline smooth loofah & annual & fall-spring & yes & yes & seed \\
\hline Chinese okra & annual & fall-spring & yes & yes & seed \\
\hline chayote tips & perennial & all year & yes & yes & whole fruit \\
\hline long squash & annual & fall-spring & sometimes & yes & seed \\
\hline tindora & semi-perennial & all year & yes & yes & stem cutting \\
\hline parvar & semi-perennial & all year & yes & yes & stem cutting; dioecious \\
\hline
\end{tabular}


Table 5. Common and scientific names, synonyms, and edible portions of Asian legumes.

\begin{tabular}{||llll||}
\hline Common Name & Scientific Name & Synonyms & Edible Portion ${ }^{1}$ \\
\hline \hline Iong bean & $\begin{array}{l}\text { Vigna unguiculata (L.) Walp. } \\
\text { subsp. unguiculata (L.) }\end{array}$ & asparagus bean, yard-long bean & immature pods, immature seed \\
lablab bean & Lablab purpurus (L.) Sweet. & $\begin{array}{l}\text { hyacinth, Indian, lubia, or } \\
\text { seim bean, papadi }\end{array}$ & immature pods, immature seed \\
winged bean & $\begin{array}{l}\text { Psophocarpus tetragonolobus } \\
\text { (L.) DC }\end{array}$ & Goa bean & immature pods / immature seed, \\
guar & Cyamopsis tetragonoloba & cluster bean & leaves, root \\
& (L.) Taub. & & \\
\hline \hline
\end{tabular}

Table 6. Target $\mathrm{pH}$, and recommended $\mathrm{N}, \mathrm{P}_{2} \mathrm{O}_{5}$, and $\mathrm{K}_{2} \mathrm{O}$ fertilizer for specialty Asian legumes.

\begin{tabular}{|c|c|c|c|c|c|c|c|c|c|c|c|}
\hline \multirow[t]{2}{*}{ Target pH } & $\mathrm{N} \mathrm{Ib/A}$ & VL & $\mathbf{L}$ & M & H & VH & VL & $\mathbf{L}$ & M & H & VH \\
\hline & & \multicolumn{7}{|c|}{ (Ib/A/crop season) } & \multicolumn{3}{|c|}{$\mathrm{K}_{2} \mathrm{O}$} \\
\hline \multicolumn{12}{|c|}{ long bean, lablab bean, winged bean, guar } \\
\hline 6.5 & 90 & 120 & 100 & 80 & 0 & 0 & 120 & 100 & 80 & 0 & 0 \\
\hline
\end{tabular}

Table 7. Life cycle, season, growth habit, mulching, and planting material for Asian legumes.

\begin{tabular}{|llllll||}
\hline Crop & Life-cycle & Season & $\begin{array}{l}\text { Growth } \\
\text { Habit }\end{array}$ & Mulch & $\begin{array}{l}\text { Planting } \\
\text { Material }\end{array}$ \\
\hline \hline long bean - bush & annual & fall-spring & determinate & no & seed \\
long bean - pole & annual & fall-spring & indeterminate & yes & seed \\
lablab bean - bush & perennial grown as an annual & fall-spring & determinate & no & seed \\
lablab bean - pole & perennial grown as an annual & fall-spring & indeterminate & yes & seed \\
winged bean & perennial grown as an annual & all year & indeterminate & yes & scarified seed \\
guar & annual & fall-spring & determinate & yes & seed \\
\hline
\end{tabular}

Table 8. Common and scientific names, synonyms, and edible portions of Asian solanums.

\begin{tabular}{|llll||}
\hline Common Name & Scientific Name & Synonyms & Edible Portion \\
\hline \hline Thai eggplant & Solanum melonogena L. & & immature fruit \\
Japanese eggplant & Solanum melonogena L. & Chinese eggplant & immature fruit \\
pea eggplant & Solanum torvum Swartz & cherry eggplant & immature fruit, tender shoots \\
bird pepper & Capsicum frutescens L. & & immature or mature fruit \\
\hline \hline${ }^{1}$ Italics indicate the edible portion grown in South Florida & & \\
\hline
\end{tabular}

Table 9. Target $\mathrm{pH}$, and recommended $\mathrm{N}_{2} \mathrm{P}_{2} \mathrm{O}_{5}$, , and $\mathrm{K}_{2} \mathrm{O}$ fertilizer for specialty Asian solanums.

\begin{tabular}{|c|c|c|c|c|c|c|c|c|c|c|c|}
\hline \multirow[t]{2}{*}{ Target pH } & $\mathrm{N} \mathrm{lb} / \mathrm{A}$ & VL & $\mathbf{L}$ & M & H & VH & VL & $\mathbf{L}$ & M & H & VH \\
\hline & & \multicolumn{4}{|c|}{$\mathrm{P}_{2} \mathrm{O}_{5}$} & $(\mathrm{Ib} / \mathrm{A} / \mathrm{s}$ & \multicolumn{5}{|c|}{$\mathrm{K}_{2} \mathrm{O}$} \\
\hline \multicolumn{12}{|c|}{ Thai, Japanese \& pea eggplant } \\
\hline & 160 & 160 & 130 & 100 & 0 & 0 & 160 & 130 & 100 & 0 & 0 \\
\hline 6.5 & 175 & 160 & 130 & 100 & 0 & 0 & 160 & 130 & 100 & 0 & 0 \\
\hline
\end{tabular}


Table 10. Injection schedule for $\mathrm{N}$ and $\mathrm{K}$ for specialty Asian solanaceous crops grown on soils testing very low in $\mathrm{K}$.

\begin{tabular}{|c|c|c|c|c|c|c|}
\hline \multirow[b]{2}{*}{ Crop } & \multicolumn{2}{|c|}{ Total nutrients $(\mathrm{Ib} / \mathrm{A})$} & \multicolumn{2}{|c|}{ Crop development } & \multicolumn{2}{|c|}{ Injection (Ib/A/day) } \\
\hline & $\mathbf{N}$ & $\mathrm{K}_{2} \mathrm{O}$ & Stage & Weeks & $\mathbf{N}$ & $\mathrm{K}_{2} \mathrm{O}$ \\
\hline Thai, Japanese & 160 & 160 & 1 & 2 & 1.0 & 1.0 \\
\hline \multirow[t]{3}{*}{ \& pea eggplant } & & & 2 & 2 & 1.5 & 1.5 \\
\hline & & & 3 & 6 & 2.5 & 2.0 \\
\hline & & & 4 & 3 & 1.5 & 1.5 \\
\hline \multirow[t]{5}{*}{ bird's eye pepper } & 175 & 160 & 1 & 2 & 1.0 & 1.0 \\
\hline & & & 2 & 2 & 1.5 & 1.5 \\
\hline & & & 3 & 7 & 2.5 & 2.5 \\
\hline & & & 4 & 1 & 1.5 & 1.5 \\
\hline & & & 5 & 1 & 1.0 & 1.0 \\
\hline
\end{tabular}

Table 11. Life cycle, season, mulching, and planting material for Asian solanums.

\begin{tabular}{|lllll||}
\hline Crop & Life-cycle & Season & Mulch & Planting Material \\
\hline \hline Thai eggplant & annual to semi-perennial & fall-spring & yes & seed \\
Japanese eggplant & annual & fall-spring & yes & seed \\
pea eggplant & semi-perennial & all year & yes & seed / stem cutting \\
bird pepper & annual to semi-perennial & fall-spring & yes & seed \\
\hline
\end{tabular}

Table 12. Common and scientific names, synonyms, and edible portions of other Asian vegetables.

\begin{tabular}{|llll||}
\hline Common Name & Scientific Name & Synonyms & Edible Portion ${ }^{1}$ \\
\hline \hline jute & Corchorus olitorius L. & Jews' marrow, bush okra & immature fruit \\
callaloo & Colocasia esculenta (L.) Schott & taro leaves & young leaves \\
callaloo & Amaranthus Spp. & edible amaranth & tender shoots, leaves, sprouted seeds \\
sweetpotato & Ipomoea batatas (L.) Lam. & & stem tips \\
\hline \hline 1 Italics indicate the edible portion grown in South Florida & & \\
\hline \hline
\end{tabular}

Table 13. Life cycle, season, and planting material for other Asian vegetables.

\begin{tabular}{|llll||}
\hline Crop & Life-cycle & Season & Planting Material \\
\hline \hline jute & annual or short lived perennial & fall-spring to all year & seed \\
callalo0 & perennial & all year & corm \\
callalo0 & annual & fall-spring & seed \\
sweetpotato tops & perennial & all year & stem cutting or tubers \\
\hline
\end{tabular}

Table 14. Average yield and price for selected Asian vegetables in Miami-Dade County (1988-89 \& 1995-96).

\begin{tabular}{||lccc||}
\hline Crop & Yield Lbs/acre & \multicolumn{2}{c||}{ Average Price/lb } \\
& & $\mathbf{1 9 8 8 - 8 9}$ & $\mathbf{1 9 9 5 - 9 6}$ \\
\hline \hline bitter melon & 3,200 & $\$ 1.44$ & $\$ 1.64$ \\
Chinese okra & 4,500 & n.a. & n.a. \\
fuzzy squash & 13,500 & n.a. & n.a. \\
long beans & 4,200 & $\$ 1.25$ & $\$ 1.38$ \\
long squash & 13,500 & $\$ 2.25$ & n.a. \\
smooth loofah & 2,400 & $\$ 1.28$ & n.a. \\
Thai \& Chinese eggplant & 6,000 & $\$ 1.82$ & $\$ 1.40$ \\
tindora & 8,500 & $\$ 1.00$ & $\$ 1.00$ \\
winged bean & 2,400 & $\$ 1.00$ & n.a. \\
\hline
\end{tabular}

